$40: 25$ up to $52: 13$ can be obtained with the exceptions of $50: \mathrm{I}_{5}$ and $5 \mathrm{I}: \mathrm{I}_{4}$ corresponding to $28: 37$ and $27: 38$. But none of these squares can be of the types of Figs. I and 2. The remaining figures give a square of these different proportions, and I have tried to illustrate some of the different possible positions of a heart in a square of fifth order.

Thus the number of "Bordered Squares and their
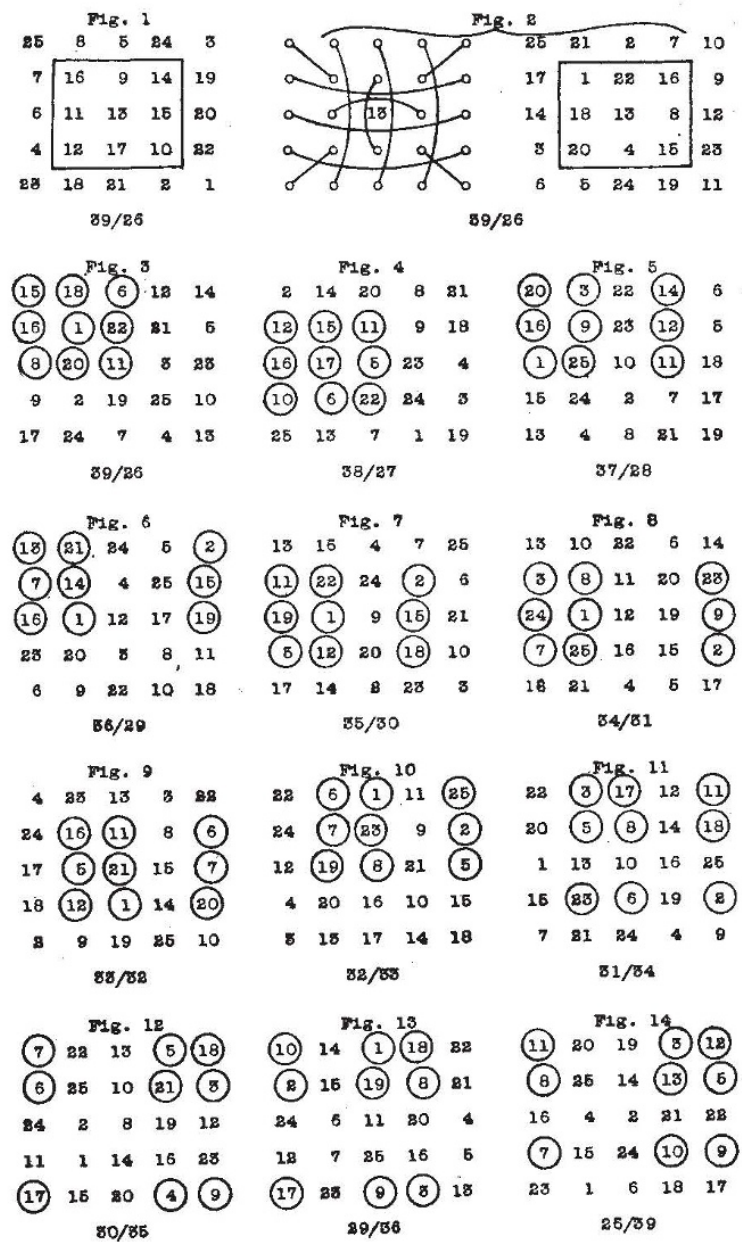

Derivatives" is very great, and if other known squares are included, such as pandiagonal squares, associated squares and their variations, rectangle squares, etc., the grand total of magic squares of fifth order, the 5 rows and 5 columns and 2 diagonals of which are magic, easily exceeds $\mathrm{I}, 000,000$. I have accounted for more than a million myself, and that is without the many squares indicated above as unknown, the enumeration of which would be very laborious and intricate. J. C. Burnett.

Barkston, near Grantham, Lincs.

\section{On the Spark Spectrum of Lithium.}

IN a letter to the editor of this journal (NATURE, Feb. 7, I925, P. I9I) an account was given of a method for excitation of spark spectra, by which a vapour jet of the substance under examination is exposed to bombardment by electrons from an incandescent wire. During the development of the apparatus certain modifications were introduced with the result that a very intense source of light has been obtained. Under ordinary conditions the current through the discharge chamber is of the order of $I$ amp. and can be maintained during several hours. It has furthermore been possible to work with this discharge apparatus in connexion with a vacuum spectrograph manufactured by Adam Hilger, Ltd., in such a way that no fluorite windows are introduced in the path of light.

With this apparatus the investigation of the spark spectrum of lithium described in the former note has been continued, and it has especially been possible to extend the classification of the spectrum denoted by Schüler as the doublet system of the Li II spectrum by inclusion of a number of further lines. Table I. gives a survey of the hitherto observed lines belonging to this system. The lines marked with an asterisk were not given in my former letter. An estimate of the intensities of the lines, as they appear on the plates, is added in brackets.

\section{TABLE I.}

$\begin{array}{ll}5484 \cdot 7(8) & 2 s-2 p \\ 488 \mathrm{I} \cdot 3\left(2 \frac{1}{2}\right) & 3 p-4 s \\ 467 \mathrm{x} \cdot 8\left(3 \frac{1}{2}\right) & 3 d-4 f \\ 4325 \cdot 7(3) & 3 p-4 d \\ 3684 \cdot \mathbf{I}(2) & 3 s-4 p \\ 3 \mathbf{I} 95 \cdot 8(3) & 3 d-5 f \\ 3 \mathrm{I} 55 \cdot 4(2) & 3 p-5 s \\ 3029 \cdot \mathbf{I}\left(2 \frac{1}{2}\right) & 3 p-5 d \\ 2728 \cdot 4(2) & 3 d-6 f \\ * 2674 \cdot 4(2) & 3 s-5 p\end{array}$

$* 2657 \cdot 3\left(\mathrm{I} \frac{1}{2}\right) 3 p-6 s$ *2605. I ( $\left.1 \frac{1}{2}\right) 3 p-6 d$ $*_{2507} \circ$ ( (1 $\left.\frac{1}{2}\right) 3 d-7 f$ $*_{2430 \cdot 0}$ (I) $3 p-75$

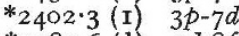
$*_{23} 8 \mathrm{r} \cdot 6\left(\frac{1}{2}\right) \quad 3 d-8 f$ *2330.0 ( $\left.\frac{1}{2}\right) \quad 3 s-6 p$ * r653.3 (6) $2 p-3 s$ *I493. I (4) $2 p-3 d$ *I198.0 (5) $2 s-3 p$

TABLE II.

$$
\begin{aligned}
& 2 s=\mathrm{r} 34,033(n *=\mathrm{x} \cdot 8 \mathrm{Io}) \\
& 3^{s}=55,3 \times 8\left(n^{*}=2 \cdot 8 \times 7\right) \\
& 4 s=30,097\left(n^{*}=3 \cdot 819\right) \\
& 5 s=18,895\left(n^{*}=4.820\right) \\
& 6 s=12,957\left(n^{*}=5.820\right) \\
& 7 s=9,438\left(n^{*}=6.819\right) \\
& 2 p=\operatorname{II}_{5}, 806(n *=\mathrm{I} \cdot 947) \\
& 3 p=50,578\left(n^{*}=2 \cdot 946\right) \\
& 4 p=28,182\left(n^{*}=3.946\right) \\
& 5 p=17,938\left(n^{*}=4.946\right) \\
& 6 p=12,4 \text { I } 3\left(n^{*}=5 \cdot 946\right) \\
& 3^{d}=48,834\left(n^{*}=2 \cdot 998\right) \\
& 4 d=27,467(n *=3.997) \\
& 5 d=\mathrm{I} 7,574(n *=4.997) \\
& 6 d=\mathrm{r} 2,203\left(n^{*}=5 \cdot 997\right) \\
& 7 d=8,964(n *=6 \cdot 997) \\
& 4 f=27,435\left(n^{*}=4^{\cdot 000}\right) \\
& 5 f=17,55^{2}\left(n^{*}=5 \cdot 000\right) \\
& 6 f=12,193(n *=6.000) \\
& 7 f=8,958\left(n^{*}=7 \cdot 000\right)
\end{aligned}
$$

In Table II. the scheme of terms corresponding to this spectrum has been given, together with the effective quantum numbers $\left(n^{*}\right)$.

It will be seen that the conclusions drawn in the former letter regarding the doublet spectrum are at all points confirmed. As regards the singlet system of $\mathrm{Li}$ II a few further lines in the far ultraviolet have been observed, and at the same time it has been found that several of the lines marked with an interrogation sign in Table I. of my former letter are due to impurities. It is hoped in the near future to bring a more complete term scheme of this spectrum.

\section{Universitetets Institut for teoretisk Fysik, Copenhagen, September ro.}

\section{Spermatogenesis in a Spider (Amaurobius sp.).}

AT the suggestion of Prof. J. Brontë Gatenby, I recently made some preparations of spider's testis, in order, if possible, to test the accuracy of the observations described by Prof. Ernest Warren in a letter to NATURE of September $\mathrm{x} 2$. In my preparations I have found many of the appearances figured by Prof. Warren. Fig. I, probably corresponding to Prof. Warren's Fig. 3, is an early prophase of the first spermatocyte division; the clumps of chromatin are the first appearance of the chromosomes, and one No. 2920 , vOL. I 16 ] 